\title{
Multitarget stool DNA test compared with fecal occult blood test for colorectal cancer screening
}

\author{
CUIPING YANG ${ }^{1 *}$, WEI WU ${ }^{2 *}$, YANPING YANG ${ }^{3 *}$, XIAOJIN YANG ${ }^{4 *}$, JING SUN $^{2 *}$, WEIYU ZHANG ${ }^{5^{*}}$,

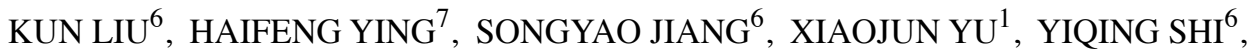 \\ YUFEN ZHOU $^{1}$, SHIYAN ZHU ${ }^{1}$, YING XU ${ }^{1}$, YANFEI DING ${ }^{1}$, LING XIE ${ }^{1}$, BOER CAI $^{8}$, \\ XIAORONG XIN $^{1}$, PING CHEN ${ }^{1}$, REN ZHAO $^{6}$ and YUNLIN WU ${ }^{1}$ \\ ${ }^{1}$ Department of Gastroenterology, Shanghai Jiao Tong University, School of Medicine, \\ Ruijin Hospital North, Shanghai 201800; ${ }^{2}$ Department of Gastroenterology, Shanghai Jiao Tong University, School of \\ Medicine, Ruijin Hospital, Shanghai 200025; ${ }^{3}$ Department of Laboratory, Jiujiang Maternity and Child Health Hospital; \\ ${ }^{4}$ Department of Infection Control, Jiujiang No. 1 People's Hospital, Jiujiang, Jiangxi 332000; \\ ${ }^{5}$ Department of Traditional Chinese Medicine, Shanghai Jiao Tong University, School of Medicine, \\ Ruijin Hospital North, Shanghai 200025; Departments of ${ }^{6}$ General Surgery and ${ }^{7}$ Traditional Chinese Medicine; \\ ${ }^{8}$ Nursing Department of Gastroenterology, Shanghai Jiao Tong University, School of Medicine, \\ Ruijin Hospital North, Shanghai 201800, P.R. China
}

Received October 15, 2019; Accepted April 23, 2020

DOI: $10.3892 / \mathrm{ol} .2020 .11674$

\begin{abstract}
Patient screening is important for early diagnosis of colorectal cancer (CRC). The present study aimed to compare the multitarget stool DNA (mt-sDNA) test with the fecal occult blood test (FOBT) for CRC screening. A total of 151 individuals were screened using colonoscopy, mt-sDNA and FOBT for the detection of CRC and adenoma. The results of the mt-sDNA test and FOBT were compared with colonoscopy to examine their sensitivity and specificity. Subsequently, the sensitivity and specificity of the mt-sDNA test were compared with those of FOBT in CRC and large adenoma. Stool samples were collected from patients with CRC $(n=50)$ or large adenoma $(n=51)$, as well as from normal controls $(n=50)$. The mt-sDNA test outperformed FOBT in detecting CRC with a sensitivity of $90.0 \%$ (45/50) vs. $42.0 \%$ (21/50), advanced adenoma with a sensitivity of $70.6 \%(36 / 51)$ vs. $19.6 \%$ (10/51), stage I-III CRC with a
\end{abstract}

Correspondence to: Dr Ping Chen, Department of Gastroenterology, Shanghai Jiao Tong University, School of Medicine, Ruijin Hospital North, 999 Xiwang Road, Jiading, Shanghai 201800, P.R. China

E-mail: dr_chenping2020@163.com

Dr Ren Zhao, Department of General Surgery, Shanghai Jiao Tong University, School of Medicine, Ruijin Hospital North, 999 Xiwang Road, Jiading, Shanghai 201800, P.R. China

E-mail: zhaorensurgeon@aliyun.com

${ }^{*}$ Contributed equally

Key words: multitarget stool DNA, adenoma, colorectal cancer, sensitivity, specificity sensitivity of $91.9 \%$ (34/37) vs. $29.7 \%$ (11/37), and stage IV CRC with a sensitivity of $84.6 \%(11 / 13)$ vs. $76.9 \%(10 / 13)$. In addition, the mt-sDNA test exhibited a specificity of $94.0 \%(47 / 50)$ in detecting CRC, which was superior to FOBT with a specificity of $90.0 \%$ (45/50). Therefore, the mt-sDNA test may have higher sensitivity and specificity compared with FOBT in diagnosing both CRC and advanced adenoma.

\section{Introduction}

Colorectal cancer (CRC) is a common malignancy associated with mutations in multiple genes, such as KRAS (1) and $S D C 2$ (2). Progression from a benign tumor to CRC takes up to 10 years in $80 \%$ of affected individuals (3). Therefore, CRC screening is critical for early detection and treatment of CRC. The fecal occult blood test (FOBT) and colonoscopy are the mainstay of CRC screening. However, the FOBT has a low diagnostic performance, particularly for colorectal adenoma (3-5). Colonoscopy is the gold standard for diagnosing CRC with good sensitivity and specificity; however, it is associated with a high risk of complications and low compliance (5). Cancer cells from early-stage CRC are continuously shed into the colonic lumen and mixed into stool (6). Tests for genetic and epigenetic alterations in fecal DNA have been considered as a possible method for the early detection of CRC (7).

$K R A S$ is a common oncogene in malignant tumors. KRAS mutations are detected in $30-40 \%$ of CRCs (1). There are seven mutation hotspots that account for $>90 \%$ of the KRAS mutations, including Gly12Asp, Gly12Val, Gly12Ser, Gly12Cys, Gly12Ala, Gly12Arg and Gly13Asp (8). It has been demonstrated that mutations in codons 12 and 13 of exon 2 of KRAS are closely associated with the development of CRC, and mutations in codon 12 are associated with a less favorable prognosis compared with mutations in codon 13 (9). 
Hypermethylation of $\mathrm{CpG}$ islands in gene promoter regions suppresses specific gene expression and promotes tumorigenesis in various types of cancer (1). The early occurrence of $\mathrm{CRC}$ is closely associated with the methylation of CRC-related gene promoter regions (10-12). The $\mathrm{N}$-myc downstream-regulated gene (NDRG) 4 is a member of the NDRG family of tumor suppressor genes (13). It has been demonstrated that the 5 ' regulatory region of $N D R G 4$ contains $\mathrm{CpG}$ islands, which are often methylated during the development of CRC (13). Methylation of $N D R G 4$ is considered to be an important biological characteristic of CRC (14). Therefore, $N D R G 4$ may be a potential diagnostic biomarker for CRC screening.

Syndecan-2 (SDC2), also known as fibroglycan, encodes a transmembrane (type I) heparan sulfate proteoglycan and regulates adhesion and proliferation of colon carcinoma cells (15). Hypermethylation of $S D C 2$ has been detected at high frequency in the blood of patients with CRC (2). As a molecular marker of potential CRC, SDC2 methylation demonstrates a high degree of specificity for the diagnosis of early-stage tumors (16).

The tissue factor pathway inhibitor (TFPI) 2 belongs to a previously described group in embryonic cells of polycomb group-marked genes that may be predisposed to aberrant DNA methylation in the early stages of CRC carcinogenesis (17). It has been demonstrated that TFPI2 levels determined by fecal DNA testing are associated with CRC recurrence and early-stage CRC (17-23).

In the present study, a multitarget stool DNA (mt-sDNA) test was designed, including quantitative molecular assays for KRAS mutations and aberrant NDRG4, SDC2 and TFPI2 methylation for the diagnosis of CRC. The diagnostic performance of the mt-sDNA test was compared with a commercially available FOBT in the detection of carcinoma and large adenoma ( $\geq 1 \mathrm{~cm}$ in diameter).

\section{Materials and methods}

Participants and stool collection. The present study included 151 participants who underwent colonoscopy at Shanghai Jiao Tong University, School of Medicine, Ruijin Hospital North (Shanghai, China) between January 2016 and January 2017. The inclusion criteria were as follows: i) Colorectal adenoma ( $\geq 1 \mathrm{~cm}$ in diameter; smaller or diminutive polyps excluded); or ii) colorectal carcinoma; and iii) age $>18$ years. Patients with the following conditions were excluded: i) Contraindications to colonoscopy; ii) severe gastrointestinal bleeding; and iii) hemorrhoids. A total of 50 participants who were free of colorectal polyps or tumors were selected from individuals who were receiving routine medical examinations.

Stool was collected from all participants prior to bowel purgation and colonoscopy, or otherwise 1 week after the colonoscopy but prior to neoplasm resection. The FOBT was performed before addition of the preservative buffer to the stool. The homogenized stools were stored at $-20^{\circ} \mathrm{C}$ for the subsequent mt-sDNA test. Colorectal adenoma or carcinoma tissues and adjacent normal tissues within $1 \mathrm{~cm}$ of the tumor were biopsied and stored in $-196^{\circ} \mathrm{C}$ liquid nitrogen prior to DNA extraction.

FOBT. The FOBT was performed using a guaiac-based immunochemical kit according to the manufacturer's protocol
(Hemosure T1-CK50, W.H.P.M. Bioresearch \& Technology) with a minimum detection limit of $0.2 \mu \mathrm{g} / \mathrm{ml}$. Depending on whether a large adenoma or tumor was identified during colonoscopy, the test results were classified as follows: A positive result was a true positive if a neoplasm was detected, or a false positive if no neoplasm was detected; a negative result was a false negative if a neoplasm was detected, or a true negative if no neoplasm was detected. Sensitivity and specificity were expressed as percentages.

DNA extraction. Frozen or fresh colorectal tissues were collected from 50 patients with CRC and 51 patients with colorectal adenoma, and were used to determine whether mutated KRAS and hypermethylated NDRG4, SDC2 and TFPI2 could be used to detect CRC and large adenoma. DNA was extracted using a TIANamp DNA kit (Tiangen Biotech Co., Ltd.) according to the manufacturer's protocol. The methylation levels of NDRG4,SDC2 and TFPI2 were detected using quantitative (q)PCR.

Stool samples were thawed at room temperature and homogenized. The aliquots were transferred to tubes and centrifuged at $14,000 \times \mathrm{g}$ for $5 \mathrm{~min}$ at room temperature. The supernatant was used as the source of mt-sDNA. The mt-sDNA markers were enriched using sequence-specific DNA captures and magnetic beads-based oligonucleotides, and purified using magnetic separation.

Bisulfite treatment. The tissue DNA and stool DNA were treated with bisulfite using an EZ DNA methylation kit (Zymo Research Corp.) according to the manufacturer's protocol. For tissue DNA, $600 \mathrm{ng}$ genomic DNA was added into the bisulfite reaction and eluted in $30 \mu \mathrm{l}$ Tris/EDTA (TE) buffer. For stool DNA, $30 \mu 1$ captured DNA was added into the reaction and eluted in $20 \mu \mathrm{l}$ TE buffer.

Methylation-specific qPCR. The test panel included three methylated (m) genes ( $m N D R G 4, m S D C 2$ and $m T F P I 2)$. The mutant forms of KRAS and $\beta$-actin were used as references. The mt-sDNA for the methylation assay was treated with bisulfite. The genomic DNA was used for the KRAS mutation assay. Multiplex qPCR was used to detect the mutation and methylation profile of candidate genes (ABI 7500 Real-Time PCR system; Applied Biosystems; Thermo Fisher Scientific, Inc.). Each run consisted of sDNA samples, positive controls ( $m N D R G 4, m S D C 2, m T F P I 2$, mutant KRAS and internal control), and negative controls (water blanks). Briefly, each multiplex PCR assay was performed with a final reaction mixture volume of $20 \mu 1$ containing $0.5 \mathrm{U}$ Phusion polymerase (Thermo Fisher Scientific, Inc.) in high-fidelity Phusion buffer with a final concentration of $200 \mu \mathrm{M}$ deoxynucleotide triphosphates and $3 \mathrm{mM} \mathrm{MgCl}$. The primers were used at a final concentration of 0.2-0.4 $\mu \mathrm{M}$. Primer sequences are listed in Table I. SYBR-Green I (Cambrex Bio Science Rockland, Inc.) was diluted as recommended by the manufacturer. The hot-start technique was used to prevent non-specific amplification (24). The amplification cycles consisted of incubation at $98^{\circ} \mathrm{C}$ for $30 \mathrm{sec}, 65^{\circ} \mathrm{C}$ for $30 \mathrm{sec}, 72^{\circ} \mathrm{C}$ for $30 \mathrm{sec}$ and $72^{\circ} \mathrm{C}$ for $10 \mathrm{sec}$. After 30 cycles, a melting curve was determined using SYBR-Green fluorescence with a ramp speed of $0.2^{\circ} \mathrm{C} / \mathrm{sec}$ between 72 and $98^{\circ} \mathrm{C}$, 
Table I. Primer sequence for the DNA methylation and $K R A S$ mutation assays.

Name

NDRG4

TFPI2

$S D C 2$

KRAS

$\beta$-actin
Primer sequences

\author{
MS: 5'-TTTAGGTTCGGTATCGTTTCGC-3' \\ 3'-CGAACTAAAAACGATACGCCG-5' \\ US: 5'-GATTAGTTTTAGGTTTGGTATTGTTTTGT-3' \\ 3'-AAAACCAAACTAAAAACAATACACCA-5' \\ MS: ATTTTTTAGGTTTCGTTTCGGC \\ 5'-GCCTAACGAAAAAAAATACGCG-3' \\ US: TTAGTTATTTTTTAGGTTTTGTTTTGGT \\ 3'-AAAAACACCTAACAAAAAAAAATACACA-5' \\ MS: 5'-AAAGATTCGGCGACCACCGAACGACTCAAACTCGAAAACTCG-3' \\ 3'-GACTCAAACTCGAAAACTCGAA-5' \\ US: 5'-TTCGGGGCGTAGTTGCGGGCGG-3' \\ 3'-TTCGGGGCGTAGTTGCGGGCGG-5' \\ 5'-CTGGTGCAGTATTTGATAGTGTA-3' \\ 3'-TGAAAATGGTCAGAGAAACCTTTA-5' \\ 5'-GCTAAGTGTGCTGGGGTCTTGGGAT-3' \\ 3'-GCTCTTTTTCTGGTGTTTGTCTCTC-5'
}

NDRG4, N-myc downstream-regulated gene 4; SDC2; syndecan-2; TFPI2, tissue factor pathway inhibitor 2; MS, methylated sequence; US, unmethylated sequence.

with a reading every $0.2^{\circ} \mathrm{C}$. The cycle threshold $(\mathrm{Ct})$ value of each gene was used to evaluate the result of each sample using the $2^{-\Delta \Delta \mathrm{Cq}}$ method (25). All assays were performed in a blinded manner.

Statistical analysis. The strand number of each marker output from the ABI 7500 was quantified according to the $\mathrm{Ct}$ value. If there was no amplification, the maximum amplification cycle number $(\mathrm{Ct}=45)$ was considered. A logistic regression with specific boundary conditions was developed to evaluate the performance of each biomarker. The single marker cut-off was identified by a logistic regression algorithm that produced dichotomous (positive/negative) results for each sample. A threshold was defined for each marker in the mt-sDNA panel that optimally separated the tumor samples from the control samples. The logistic regression assigned a weight to each component assay result and subsequently aggregated these individual marker results to obtain a logistic score. Boundary conditions for each of the methylation and mutation markers were defined on the basis of a single value for each marker above which a positive result could be inferred. A positive result for the logistic score or a value exceeding any of the boundary conditions resulted in a positive result for the mt-sDNA test. Colonoscopy-based findings were compared with the mt-sDNA test results. Sensitivity and specificity were calculated as percentages for comparison with FOBT. Receiver operating characteristic (ROC) curves were constructed to analyze the diagnostic performances of the biomarkers.

Continuous data were presented as the mean \pm SD and compared using the one-way ANOVA test followed by Tukey's post hoc test. Categorical data were presented as percentages and compared using the $\chi^{2}$ test. All statistical analyses were performed using SPSS v21.0 (IBM Corp.). $\mathrm{P}<0.05$ was considered to indicate a statistically significant difference.

\section{Results}

Patient characteristics. To determine the performance of biomarkers (NDRG4, SDC2, TFPI2 and KRAS) in detecting CRC and adenoma, an independent tissue study was performed. A total of 211 frozen or fresh colorectal tissues, including 64 pairs of $\mathrm{CRC}$ and adjacent normal tissues (median age, 63 years; range, 43-79 years; $48.4 \%$ women) and 83 colorectal adenomas $(\geq 1 \mathrm{~cm}$ in diameter; median age, 57 years; range, 39-72; $41 \%$ women) were included in the present study. Age and sex distributions were similar between patients with CRC, patients with colorectal adenomas and normal controls (Table II). In the carcinoma and adenoma tissues, $38.0 \%(19 / 50)$ and $43.1 \%(22 / 51)$ of the neoplasms were located in the colon, respectively. The demographic and clinical characteristics of the subjects are shown in Table II.

Detection of the DNA markers. mNDRG4 was detected in $92.2 \%(59 / 64)$ of the carcinoma tissues, $63.9 \%(53 / 83)$ of the adenoma tissues and $3.1 \%(2 / 64)$ of the adjacent normal tissues (Fig. 1). $m S D C 2$ was detected in $96.9 \%(62 / 64)$ of the carcinoma tissues and $78.3 \%(65 / 83)$ of the adenoma samples, with a specificity of $95.3 \%(61 / 64)$. TTFPI2 was detected in $95.3 \%(61 / 64)$ of the carcinoma tissues and $71.1 \%(59 / 83)$ of the adenoma samples, with a specificity of $93.8 \%(60 / 64)$. KRAS mutations were detected in $45.3 \%$ (29/64) of the carcinoma samples, $25.3 \%(21 / 83)$ of the adenoma samples and $3.1 \%(2 / 64)$ of the adjacent normal tissues (Fig. 1).

ROC curves were constructed for each of the four genes (Fig. 2). When comparing the cancer tissues with the adjacent 
Table II. Clinical characteristics of the participants.

\begin{tabular}{|c|c|c|c|c|}
\hline Variable & $\mathrm{CRC}(\mathrm{n}=50)$ & Adenoma $(\mathrm{n}=51)$ & Control $(n=50)$ & P-value \\
\hline Age, years (mean $\pm S D)$ & $60.2 \pm 13.6$ & $58.5 \pm 10.7$ & $64.9 \pm 11.0$ & $>0.05$ \\
\hline Female & $52.0 \%(26 / 50)$ & $60.8 \%(31 / 51)$ & $46.0 \%(23 / 50)$ & $>0.05$ \\
\hline Colon neoplasms & $38.0 \%(19 / 50)$ & $43.1 \%(22 / 51)$ & - & $>0.05$ \\
\hline Rectum neoplasms & $62.0 \%(31 / 50)$ & $56.9 \%(29 / 51)$ & - & $>0.05$ \\
\hline
\end{tabular}

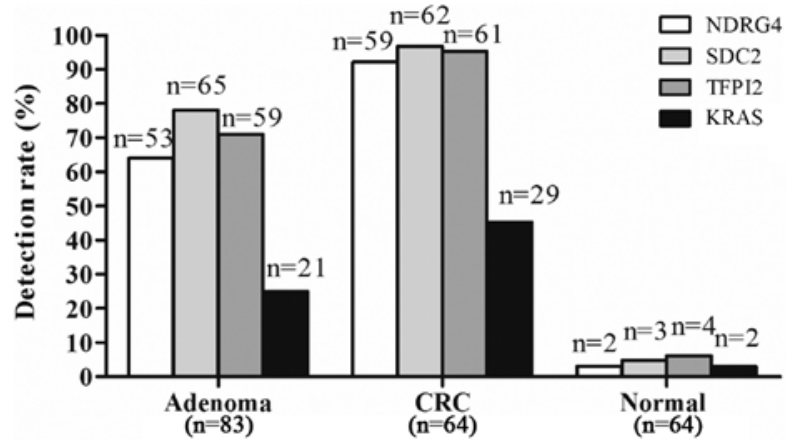

Figure 1. Detection rate of the multiple targets in adenoma, CRC and normal tissue samples. $m N D R G 4$ was detected in $92.2 \%(59 / 64)$ of the carcinoma tissues, $63.9 \%(53 / 83)$ of the adenoma tissues and $3.1 \%(2 / 64)$ of the adjacent normal tissues. $m S D C 2$ was detected in $96.9 \%(62 / 64)$ of the carcinoma tissues and $78.3 \%(65 / 83)$ of the adenoma samples, with a specificity of $95.3 \%(61 / 64)$. $m$ TFPI2 was detected in $95.3 \%(61 / 64)$ of the carcinoma tissues and $71.1 \%(59 / 83)$ of the adenoma samples, with a specificity of $93.8 \%(60 / 64)$. KRAS mutations were detected in $45.3 \%(29 / 64)$ of the carcinoma samples, $25.3 \%(21 / 83)$ of the adenoma samples and $3.1 \%(2 / 64)$ of the adjacent normal tissues. CRC, colorectal cancer; NDRG4, N-myc downstream-regulated gene 4; SDC2, syndecan-2; TFPI2, tissue factor pathway inhibitor 2 .

normal tissues, the area under the curve (AUC) values were 0.949, 0.992, 0.969 and 0.785 for NDRG4, SDC2, TFPI2 and $K R A S$, respectively. When comparing adenoma tissues with adjacent normal tissues, the AUC values were 0.837 , $0.885,0.831$ and 0.636 for NDRG4, SDC2, TFPI2 and KRAS, respectively.

Comparison of the mt-sDNA test with FOBT in CRC detection. A total of 50 patients with CRC underwent the mt-sDNA test. The sensitivity of the mt-sDNA test for CRC was $90.0 \%(45 / 50)$, with a $91.9 \%(34 / 37)$ sensitivity for stage I-III CRC and an $84.6 \%(11 / 13)$ sensitivity for stage IV CRC (Fig. 3). The FOBT had a sensitivity of $42.0 \%$ (21/50) for CRC in the same samples, with a $29.7 \%$ (11/37) sensitivity for stage I-III CRC and a 76.9\% (10/13) sensitivity for stage IV CRC (Fig. 3). These results demonstrated that the mt-sDNA test outperformed the FOBT in detecting CRC. In addition, the specificity of the mt-sDNA test $(94.0 \% ; 47 / 50)$ was higher than that of the FOBT $(90.0 \%$; 45/50) (data not shown).

Comparison of the mt-sDNA test with FOBT for the detection of large adenoma. A total of 51 individuals were diagnosed with advanced adenoma by colonoscopy. The size of the adenoma was $1-2 \mathrm{~cm}$ in 12 samples, $2-3 \mathrm{~cm}$ in 21 samples and $3-5 \mathrm{~cm}$ in 18 samples. The mt-sDNA test detected 7 adenomas of 1-2 cm, 15 adenomas of 2-3 cm and 14 adenomas of 3-5 cm, whereas FOBT detected 2 adenomas of 1-2 cm, 5 adenoma of $2-3 \mathrm{~cm}$ and 4 adenomas of $3-5 \mathrm{~cm}$ (Fig. 4). The mt-sDNA test outperformed the FOBT in detecting advanced adenomas with a sensitivity of $70.6 \%(36 / 51)$ vs. $19.6 \%$ (10/51) (data not shown).

For the mt-sDNA test, the area under the ROC curve was 0.948 (95\% CI, 0.98-1) for detecting CRC and 0.844 (95\% CI, 0.83-0.93) for detecting adenomas (Fig. 5A). For FOBT, the area under the ROC curve was 0.785 (95\% CI, 0.69-0.87) for detecting CRC and 0.636 (95\% CI, 0.53-0.74) for detecting adenoma (Fig. 5B).

Detecting CRC at different sites. In terms of tumor location, the $50 \mathrm{CRC}$ samples included 10 samples in the ascending colon, 5 samples in the transverse colon, 4 samples in the descending colon and 31 samples in the rectum. The sensitivity of the mt-sDNA test for detecting CRC was $90.0 \%(9 / 10)$ for the ascending colon, $60.0 \%(3 / 5)$ for the transverse colon, $75.0 \%$ (3/4) for the descending colon and $96.8 \%$ (30/31) for the rectum, whereas the sensitivity of FOBT for detecting CRC was 50.0\% (5/10) for the ascending colon, $20.0 \%(1 / 5)$ for the transverse colon, $25.0 \%(1 / 4)$ for the descending colon and $45.2 \%$ (14/31) for the rectum (Fig. 6). The 51 adenoma samples comprised 15 in the ascending colon, 7 in the transverse colon and 29 in the rectum. The sensitivity of the FOBT for detecting adenoma was $20.0 \%$ (3/15) for the ascending colon, $14.3 \%(1 / 7)$ for the transverse colon and $20.7 \%(6 / 29)$ for the rectum, whereas the sensitivity of the mt-sDNA test for detecting adenoma was $66.7 \%$ (10/15) for the ascending colon, $57.1 \%(4 / 7)$ for the transverse colon and $72.4 \%$ (21/29) for the rectum (Fig. 7).

\section{Discussion}

The present study demonstrated that the mt-sDNA test was superior to FOBT in detecting both CRC and large adenoma with a specificity of $93.0 \%$ vs. $91.0 \%$. The current findings suggested that the mt-sDNA test may be a feasible and promising approach for early detection of CRC. FOBT is a traditional screening tool for CRC. However, it is not widely used for CRC screening in China, partially due to its inherent low sensitivity for detecting colorectal neoplasms, particularly advanced adenomas in asymptomatic patients $(26,27)$. The present study demonstrated that FOBT had a sensitivity of $19.6 \%$ for advanced adenoma and $29.7 \%$ for stage I-III CRC. The mt-sDNA test had a $50 \%$ higher sensitivity for adenomas and a $60 \%$ higher sensitivity for stage I-III CRC. In addition, the sensitivity of the mt-sDNA test for detecting CRC was $90.0 \%(9 / 10)$ in the ascending 

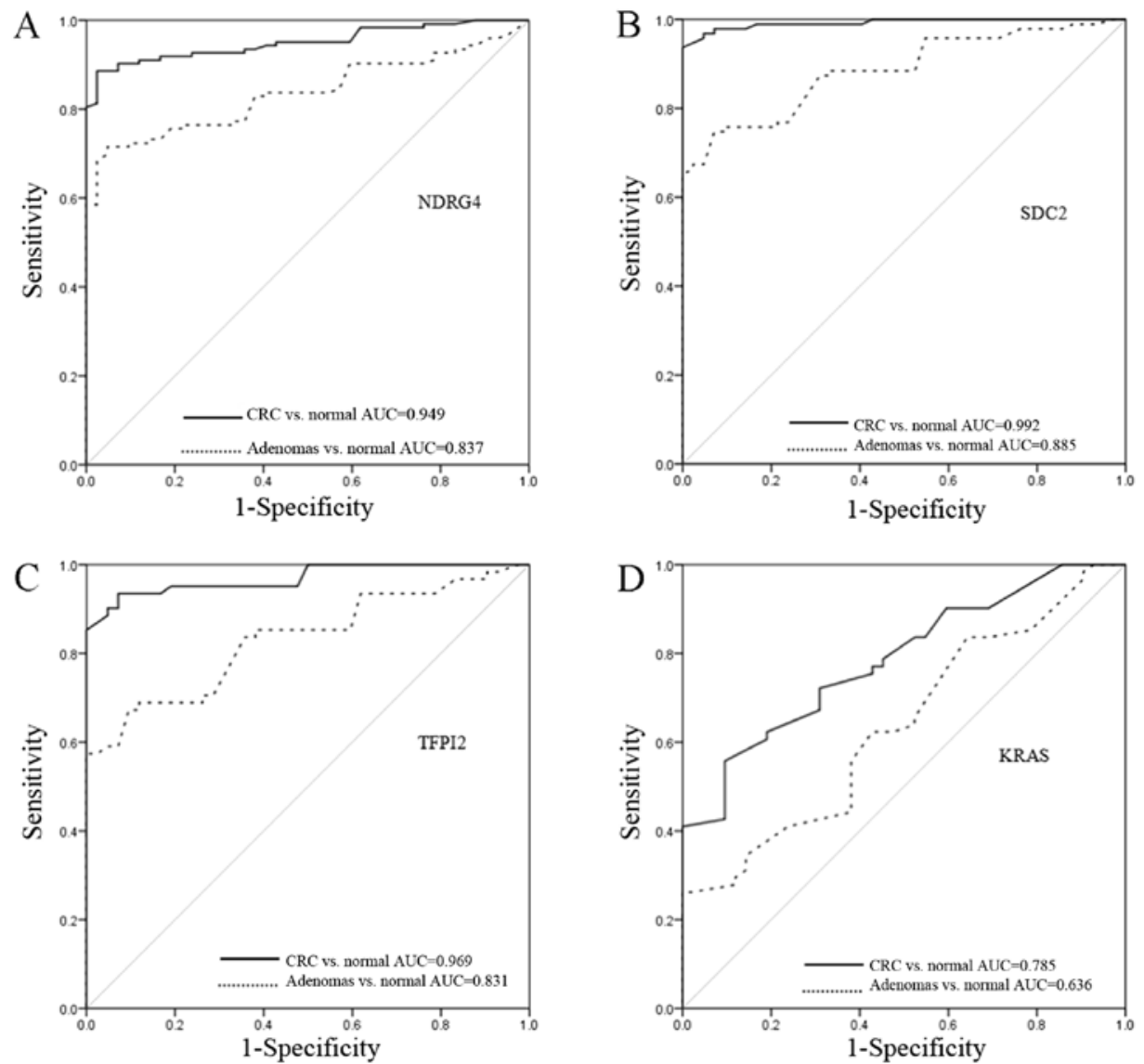

Figure 2. Receiver operating characteristic curves. Areas under the curve of (A) NDRG4, (B) SDC2 (C) TFPI2 and (D) KRAS in the carcinoma or adenoma samples were compared with normal samples. CRC, colorectal cancer; NDRG4, N-myc downstream-regulated gene 4; SDC2, syndecan-2, TFPI2, tissue factor pathway inhibitor 2; AUC, area under the curve.

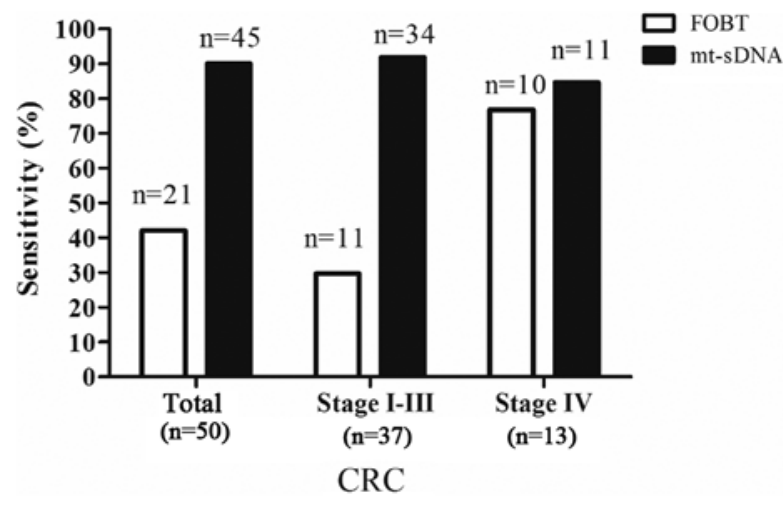

Figure 3. Sensitivity of FOBT and mt-sDNA in detecting CRC. The sensitivity of the mt-sDNA test for CRC was $90.0 \%$ (45/50), with a $91.9 \%$ (34/37) sensitivity for stage I-III CRC and an $84.6 \%$ (11/13) sensitivity for stage IV CRC. The FOBT had a sensitivity of $42.0 \%$ (21/50) for CRC in the same samples, with a $29.7 \%$ (11/37) sensitivity for stage I-III CRC and a $76.9 \%(10 / 13)$ sensitivity for stage IV CRC. FOBT, fecal occult blood test; mt-sDNA, multitarget stool DNA; CRC, colorectal cancer.

colon, $60.0 \%(3 / 5)$ in the transverse colon, $75.0 \%(3 / 4)$ in the descending colon and $96.8 \%(30 / 31)$ in the rectum. However, due to the small sample size, the current results did not support any conclusion concerning the performance of mt-sDNA for diagnosing CRC at any specific stage or location.

Colonoscopy is considered the gold standard for CRC diagnosis, but its application in CRC screening has been

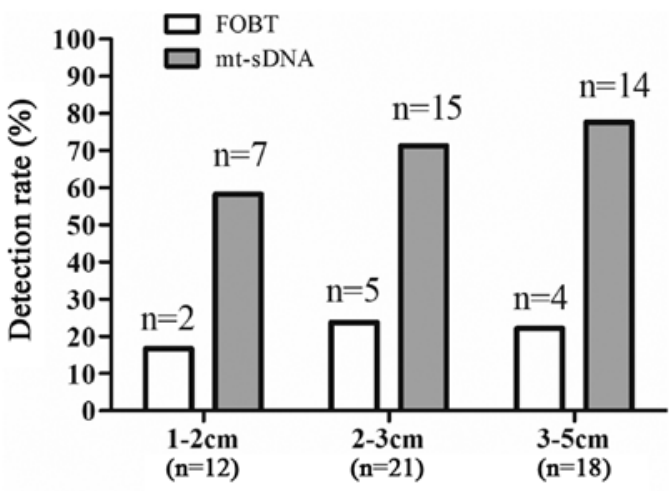

Adenoma

Figure 4. Sensitivities of the mt-sDNA test and FOBT for the detection of adenoma according to lesion size. The mt-sDNA test detected 7 adenomas of $1-2 \mathrm{~cm}, 15$ adenomas of $2-3 \mathrm{~cm}$ and 14 adenomas of $3-5 \mathrm{~cm}$, whereas FOBT detected 2 adenomas of $1-2 \mathrm{~cm}, 5$ adenoma of $2-3 \mathrm{~cm}$ and 4 adenomas of $3-5 \mathrm{~cm}$. FOBT, fecal occult blood test; mt-sDNA, multitarget stool DNA.

hindered by a number of factors, such as the requirement of a visible lesion, the risk of complications and the invasiveness of the procedure, resulting in low patient compliance (28). The novel multitarget panel presented in the current study had an improved performance compared with previous findings (29). A dozen of exfoliated markers, including mutated $K R A S$ and hypermethylated NDRG4,SDC2 and TFPI2, were 

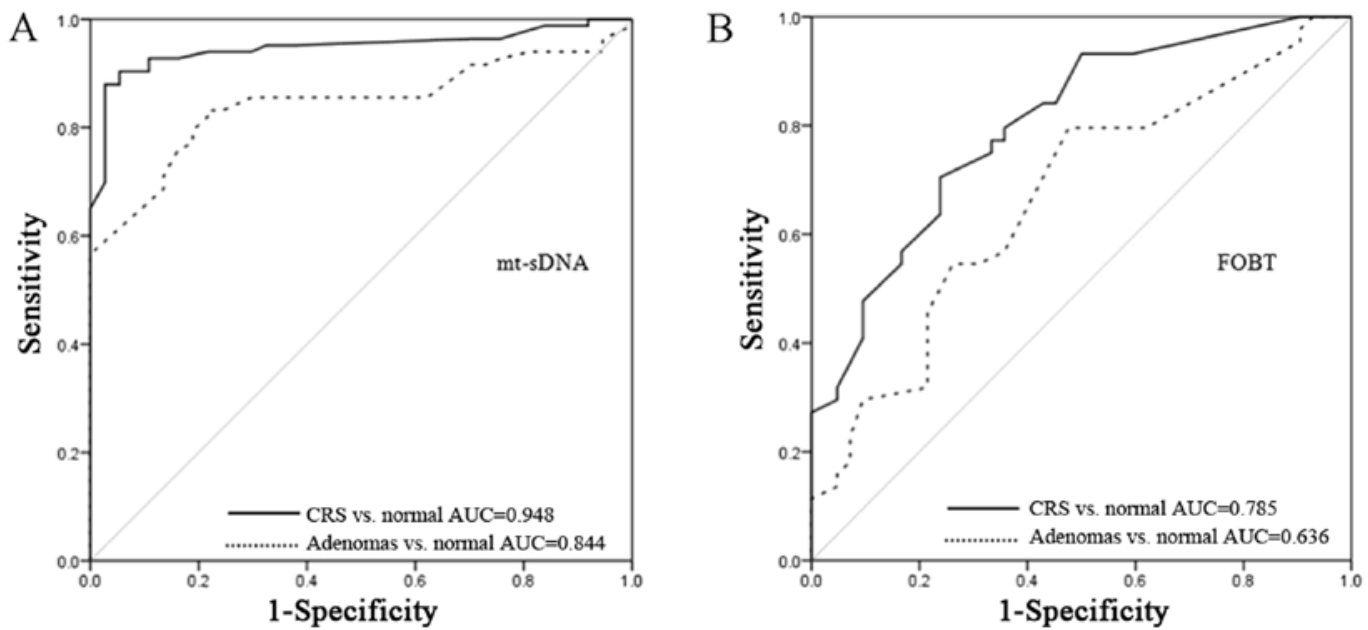

Figure 5. Receiver operating characteristic curves. Areas under the curve of (A) the mt-sDNA test and (B) FOBT for the detection of CRC and adenoma were compared with normal tissues. FOBT, fecal occult blood test; mt-sDNA, multitarget stool DNA; CRC, colorectal cancer; AUC, area under the curve.

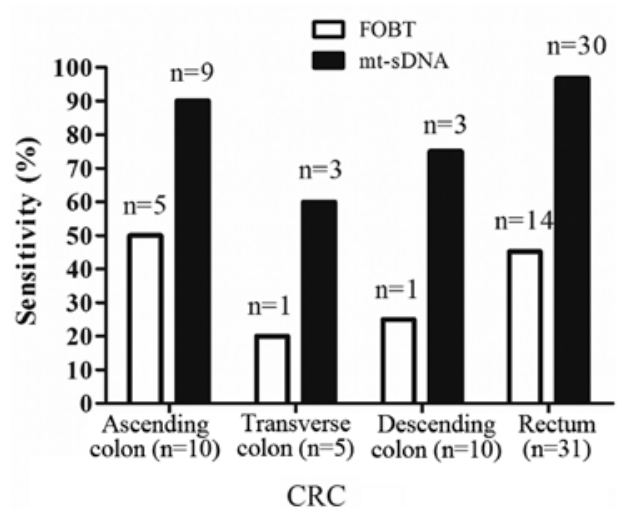

Figure 6. Sensitivities of the mt-sDNA test and FOBT for the detection of CRC according to location in the colon and rectum. The sensitivity of the mt-sDNA test for detecting CRC was $90.0 \%(9 / 10)$ for the ascending colon, $60.0 \%$ (3/5) for the transverse colon, $75.0 \%$ (3/4) for the descending colon and 96.8\% (30/31) for the rectum, whereas the sensitivity of FOBT for detecting CRC was $50.0 \%(5 / 10)$ for the ascending colon, $20.0 \%(1 / 5)$ for the transverse colon, $25.0 \%$ (1/4) for the descending colon and $45.2 \%$ (14/31) for the rectum. FOBT, fecal occult blood test; mt-sDNA, multitarget stool DNA; CRC, colorectal cancer.

analyzed in tissue and stool assays in our study. NDRG4, $S D C 2$ and TFPI2 were highly methylated in CRC tissues, distinguishing them from normal colon mucosal tissues, and KRAS mutated tumors were more likely to develop on the right side of the colon, in accordance with a previous study (30). Stool observations were consistent with the tissue assays, and the analyzed biomarkers exhibited high sensitivity and discrimination between CRC lesions and normal tissues. In the present study, the mt-sDNA panel exhibited no differences among the diverse tumor sites with $90 \%$ or higher sensitivity.

As of 2018, CRC is the third most prevalent cancer worldwide, and its morbidity and mortality in China has gradually increased (31). In the United States, the incidence and mortality of CRC have gradually decreased, mainly due to large-scale population screening, interventions for precancerous lesions for primary prevention and early detection of CRC (31). In China, screening rates for CRC remain

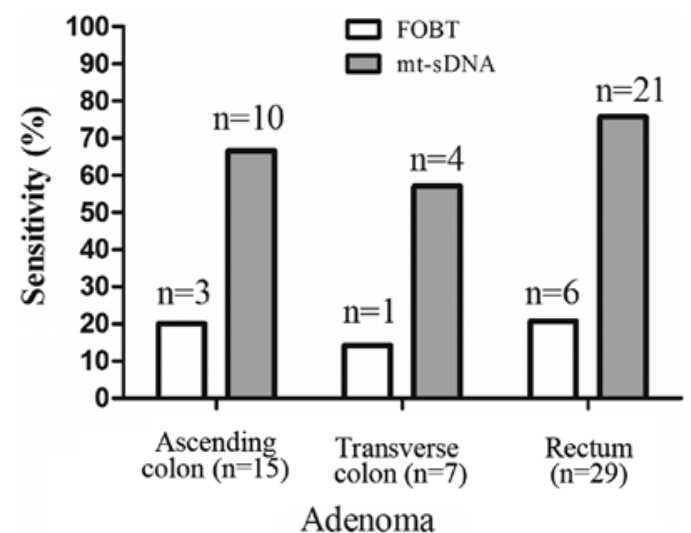

Figure 7. Sensitivities of the mt-sDNA test and FOBT for the detection of large adenoma according to location in the colon and rectum. The sensitivity of the FOBT for detecting adenoma was $20.0 \%(3 / 15)$ for the ascending colon, $14.3 \%$ (1/7) for the transverse colon and $20.7 \%$ (6/29) for the rectum, whereas the sensitivity of the mt-sDNA test for detecting adenoma was $66.7 \%(10 / 15)$ for the ascending colon, 57.1\% (4/7) for the transverse colon and $72.4 \%(21 / 29)$ for the rectum. FOBT, fecal occult blood test; mt-sDNA, multitarget stool DNA.

low and there is a shortage of medical resources. The present study offered a non-invasive approach for CRC diagnosis and screening with high sensitivity and specificity. In a previous study, a number of average-risk participants were recruited to investigate their compliance with fecal DNA testing via questionnaires, with $>90 \%$ of these individuals being prone to the mt-sDNA test, indicating that the mt-sDNA test is patient-friendly to the average-risk population (32). In addition, the mt-sDNA test may have the potential to detect CRC at an earlier stage of tumor development compared with FOBT. However, the relatively high cost of the mt-sDNA test may limit its popularity.

In conclusion, the mt-sDNA test had a higher sensitivity and specificity in diagnosing both CRC and advanced adenoma compared with FOBT. Considering its molecular diagnostic capability and its broad accessibility at clinical laboratories, the mt-sDNA test may be a valuable addition to current CRC screening options. 


\section{Acknowledgements}

Not applicable.

\section{Funding}

The present study was supported by grants from the Health and Family Planning Commission of Jiading District (grant no. 2018-KY-01) and the Shanghai Jiao Tong University School of Medicine, Ruijin Hospital North (grant no. 2018ZY16).

\section{Availability of data and materials}

The datasets used and/or analyzed during the current study are available from the corresponding author on reasonable request.

\section{Authors' contributions}

CY analyzed the samples and drafted the manuscript. WW, JS, XYa, KL, HY, YY, SJ, XYu, YS, YZ, SZ, YX, YD, LX, BC and $\mathrm{XX}$ collected the samples. PC, WZ and RZ analyzed the data and designed the study. YW conceived the study and critically revised the manuscript. All authors read and approved the final manuscript.

\section{Ethics approval and consent to participate}

The present study was approved by the Ethics Committee of Shanghai Jiao Tong University School of Medicine, Ruijin Hospital North. All participants provided written informed consent.

\section{Patient consent for publication}

All participants gave permission for publication.

\section{Competing interests}

The authors declare that they have no competing interests.

\section{References}

1. Imamura Y, Morikawa T, Liao X, Lochhead P, Kuchiba A, Yamauchi M, Qian ZR, Nishihara R, Meyerhardt JA, Haigis KM, et al: Specific mutations in KRAS codons 12 and 13, and patient prognosis in 1075 BRAF wild-type colorectal cancers. Clin Cancer Res 18: 4753-4763, 2012.

2. Xiao W, Zhao H, Dong W, Li Q, Zhu J, Li G, Zhang S and Ye M: Quantitative detection of methylated NDRG4 gene as a candidate biomarker for diagnosis of colorectal cancer. Oncol Lett 9: 1383-1387, 2015.

3. Luo SL, Hu RY, Gong WW, Wang H, Pan J, Fei FR and Yu M: Survival rate of colorectal cancer patients during 2005-2010 in Zhejiang province, China. Zhonghua Liu Xing Bing Xue Za Zhi 34: 1194-1197, 2013 (In Chinese).

4. Song LL and Li YM: Current noninvasive tests for colorectal cancer screening: An overview of colorectal cancer screening tests. World J Gastrointest Oncol 8: 793-800, 2016.

5. Iannone A, Losurdo G, Pricci M, Girardi B, Massaro A, Principi M, Barone M, Ierardi E and Di Leo A: Stool investigations for colorectal cancer screening: From occult blood test to DNA analysis. J Gastrointest Cancer 47: 143-151, 2016.
6. Ahlquist DA, Harrington JJ, Burgart LJ and Roche PC: Morphometric analysis of the 'mucocellular layer' overlying colorectal cancer and normal mucosa: Relevance to exfoliation and stool screening. Hum Pathol 31: 51-57, 2000.

7. Brenner H, Hoffmeister M, Arndt V, Stegmaier C, Altenhofen L and Haug U: Protection from right- and left-sided colorectal neoplasms after colonoscopy: Population-based study. J Natl Cancer Inst 102: 89-95, 2010.

8. Lu H, Huang S, Zhang X, Wang D, Zhang X, Yuan X, Zhang Q and Huang Z: DNA methylation analysis of SFRP2, GATA4/5, NDRG4 and VIM for the detection of colorectal cancer in fecal DNA. Oncol Lett 8: 1751-1756, 2014.

9. Renaud S, Guerrera F, Seitlinger J, Costardi L, Schaeffer M, Romain B, Mossetti C, Claire-Voegeli A, Filosso PL, Legrain M, et al: KRAS exon 2 codon 13 mutation is associated with a better prognosis than codon 12 mutation following lung metastasectomy in colorectal cancer. Oncotarget 8: 2514-2524, 2017.

10. Ross JP, Rand KN and Molloy PL: Hypomethylation of repeated DNA sequences in cancer. Epigenomics 2: 245-269, 2010.

11. Rasmussen SL, Krarup HB, Sunesen KG, Pedersen IS, Madsen PH and Thorlacius-Ussing O: Hypermethylated DNA as a biomarker for colorectal cancer: A systematic review. Colorectal Dis 18: 549-561, 2016.

12. Zou H, Harrington JJ, Shire AM, Rego RL, Wang L, Campbell ME, Oberg AL and Ahlquist DA: Highly methylated genes in colorectal neoplasia: Implications for screening. Cancer Epidemiol Biomarkers Prev 16: 2686-2696, 2007.

13. Hinoue T, Weisenberger DJ, Lange CP, Shen H, Byun HM, Van Den Berg D, Malik S, Pan F, Noushmehr H, van Dijk CM, et al: Genome-scale analysis of aberrant DNA methylation in colorectal cancer. Genome Res 22: 271-282, 2012 .

14. Mitchell SM, Ross JP, Drew HR, Ho T, Brown GS, Saunders NF, Duesing KR, Buckley MJ, Dunne R, Beetson I, et al: A panel of genes methylated with high frequency in colorectal cancer. BMC Cancer 14: 54, 2014.

15. Melotte V, Lentjes MH, van den Bosch SM, Hellebrekers DM, de Hoon JP, Wouters KA, Daenen KL, Partouns-Hendriks IE, Stessels F, Louwagie J, et al: N-Myc downstream-regulated gene 4 (NDRG4): A candidate tumor suppressor gene and potential biomarker for colorectal cancer. J Natl Cancer Inst 101: 916-927, 2009.

16. Park H, Kim Y, Lim Y, Han I and Oh ES: Syndecan-2 mediates adhesion and proliferation of colon carcinoma cells. J Biol Chem 277: 29730-29736, 2002.

17. Glöckner SC, Dhir M, Yi JM, McGarvey KE, Van Neste L, Louwagie J, Chan TA, Kleeberger W, de Bruïne AP, Smits KM, et al: Methylation of TFPI2 in stool DNA: A potential novel biomarker for the detection of colorectal cancer. Cancer Res 69: 4691-4699, 2009.

18. Oh TJ, Oh HI, Seo YY, Jeong D, Kim C, Kang HW, Han YD, Chung HC, Kim NK and An S: Feasibility of quantifying SDC2 methylation in stool DNA for early detection of colorectal cancer. Clin Epigenetics 9: 126, 2017.

19. Lao VV and Grady WM: Epigenetics and colorectal cancer. Nat Rev Gastroenterol Hepatol 8: 686-700, 2011.

20. Gerecke C, Scholtka B, Löwenstein Y, Fait I, Gottschalk U, Rogoll D, Melcher R and Kleuser B, et al: Hypermethylation of ITGA4, TFPI2 and VIMENTIN promoters is increased in inflamed colon tissue: Putative risk markers for colitis-associated cancer. J Cancer Res Clin Oncol 141: 2097-2107, 2015.

21. Kadiyska T and Nossikoff A: Stool DNA methylation assays in colorectal cancer screening. World J Gastroenterol 21: 10057-10061, 2015.

22. Zou H, Taylor WR, Harrington JJ, Hussain FT, Cao X, Loprinzi CL, Levine TR, Rex DK, Ahnen D, Knigge KL, et al: High detection rates of colorectal neoplasia by stool DNA testing with a novel digital melt curve assay. Gastroenterology 136: 459-470, 2009.

23. Ahlquist DA, Zou H, Domanico M, Mahoney DW, Yab TC, Taylor WR, Butz ML, Thibodeau SN, Rabeneck L,Paszat LF, et al: Next-generation stool DNA test accurately detects colorectal cancer and large adenomas. Gastroenterology 142: 248-256, 2012.

24. Green MR and Sambrook J: Hot start polymerase chain reaction (PCR). Cold Spring Harb Protoc 2018: 2018.

25. Livak KJ and Schmittgen TD: Analysis of relative gene expression data using real-time quantitative PCR and the 2(-Delta Delta C(T)) method. Methods 25: 402-408, 2001. 
26. Jiang X, Harrington JJ, Mahoney DW, Oberg AL, Devens ME Simonson J, Ahlquist DA and Zou H: T1102 detection of colorectal neoplasia by stool DNA testing: High discrimination with multi-marker quantitation. Gastroenterology 134 (Suppl 1): A-484, 2008

27. Huang Z, Li L and Wang J: Hypermethylation of SFRP2 as a potential marker for stool-based detection of colorectal cancer and precancerous lesions. Dig Dis Sci 52: 2287-2291, 2007.

28. Baek YH, Chang E, Kim YJ, Kim BK, Sohn JH and Park DI: Stool methylation-specific polymerase chain reaction assay for the detection of colorectal neoplasia in Korean patients. Dis Colon Rectum 52: 1452-1459, 2009.

29. Bleeker WA, Hayes VM, Karrenbeld A, Hofstra RM, Hermans J, Buys CC and Plukker JT: Impact of KRAS and TP53 mutations on survival in patients with left- and right-sided Dukes' C colon cancer. Am J Gastroenterol 95: 2953-2957, 2000.
30. Zauber AG: The impact of screening on colorectal cancer mortality and incidence: Has it really made a difference? Dig Dis Sci 60: 681-691, 2015

31. Bray F, Ferlay J, Soerjomataram I, Siegel RL, Torre LA and Jemal A: Global cancer statistics 2018: GLOBOCAN estimates of incidence and mortality worldwide for 36 cancers in 185 countries. CA Cancer J Clin 68: 394-424, 2018.

32. Yang D, Hillman SL, Harris AM, Sinicrope PS, Devens ME and Ahlquist DA: Patient perceptions of stool DNA testing for pan-digestive cancer screening: A survey questionnaire. World J Gastroenterol 20: 4972-4979, 2014.

This work is licensed under a Creative Commons Attribution-NonCommercial-NoDerivatives 4.0 International (CC BY-NC-ND 4.0) License. 